\title{
Mediating relationship of differential products in understanding integration in introductory physics
}

\author{
Nathaniel Amos and Andrew F. Heckler \\ Department of Physics, Ohio State University, 191 W. Woodruff Avenue, Columbus, Ohio 43210, USA
}

(Received 19 February 2017; revised manuscript received 4 December 2017; published 22 January 2018)

\begin{abstract}
In the context of introductory physics, we study student conceptual understanding of differentials, differential products, and integrals and possible pathways to understanding these quantities. We developed a multiple choice conceptual assessment employing a variety of physical contexts probing physical understanding of these three quantities and administered the instrument to over 1000 students in first and second semester introductory physics courses. Using a regression-based mediation analysis with conceptual understanding of integration as the dependent variable, we found evidence consistent with a simple mediation model: the relationship between differentials scores and integral scores may be mediated by the understanding of differential products. The indirect effect (a quantifiable metric of mediation) was estimated as $a b=0.29,95 \%$ CI $[0.25,0.33]$ for $N=1102$ Physics 1 students, and $a b=0.27,95 \%$ CI $[0.14,0.48]$ for $N=65$ Physics 2 students. We also find evidence that the physical context of the questions can be an important factor. These results imply that for introductory physics courses, instructional emphasis first on differentials then on differential products in a variety of contexts may in turn promote better integral understanding.
\end{abstract}

DOI: 10.1103/PhysRevPhysEducRes.14.010105

\section{INTRODUCTION}

\section{A. Physics integrals and their components}

Students enrolled in a calculus-based introductory physics course encounter tasks in which they must conceptualize, construct, and compute an integral to obtain a physical result, especially in the second semester of the standard introductory sequence. In the past few decades, research in both mathematics and physics education has demonstrated that many students struggle with such tasks. Among the earliest studies, Orton found that British students aged 16 to 22 were usually capable of computing a Riemann sum, but frequently lacked the conceptual foundation to explain its meaning or relevance to integration [1]. Further investigations have revealed that students may also have difficulty recognizing when to employ an integral [2,3], as well as determining what differential product (e.g., $F d x$ or $v d t$ ) to accumulate [4] when constructing an integral.

In order to find ways to better address these fundamental difficulties with integration, in this study we investigate students' physical and conceptual understanding of some of the basic building blocks of integration, namely, differentials and differential products, and how understanding

Published by the American Physical Society under the terms of the Creative Commons Attribution 4.0 International license. Further distribution of this work must maintain attribution to the author(s) and the published article's title, journal citation, and DOI. these concepts may lead to a physical and conceptual understanding of integrals typically encountered in physics. We begin with some justification for this approach.

\section{Differentials and differential products}

Several studies have discussed students' treatment of the differential quantity (e.g., $d x$ or $d t$ ) in both mathematical and physical contexts. In electrostatics, Nguyen and Rebello considered that a lack of physical meaning associated with the differential appeared to hinder student progress or change the meaning of the integral expression entirely [4]. Doughty et al. noted that students "may see $d x$ as [simply] a punctuation mark." For many students, the differential could be merely a notational convention to be appended on the end of the integrand. Furthermore, while analyzing students' reasoning about differentials through a series of case studies, $\mathrm{Hu}$ and Rebello identified a variety of cognitive "resources" (cf., Ref. [5]) that students may invoke while constructing physical integrals [6]. Within the context of physics problems, the most productive and widely applicable resource was that of the "small amount," in which differentials are conceptualized as very small (perhaps infinitesimally small) intervals or amounts of physical quantities.

For convenience, we will use a "process-object layered framework" for understanding physics integration. According to Sfard, some mathematical elements can be understood as both processes and (later) as structural objects [7]. As an historical example, one may examine the development of subtraction (a process), which later produced the 
concept of negative numbers (objects). This was expanded to the realm of calculus when Zandieh introduced several layers of process objects in understanding the derivative [8]. Zandieh proposed a process-object layered framework for conceptualizing derivatives, which included a ratio layer (at the most basic level, a derivative is division, a quotient), a limit layer (to shrink the denominator), and a function layer (to yield analytical functions as derivatives). In this way, each additional stage can be understood as both a verb and a noun.

Integration can be similarly dissected into process-object layers. In her development of a process-object layered framework for Riemann sums, Sealey found that students encountered the most difficulty setting up finite products analogous to the (infinitesimal) differential product of an integral [9]. Similarly, Jones found evidence that the crux of making sense of contextualized integrals (such as those in a physical scenario) may rest in the multiplicative relationship between the integrand and the differential [10]. Jones suggests that comprehending the physical meaning of a differential product as a new, "resultant," multiplicative quantity to be summed with others like it could prove more productive in physics integration, compared to more traditional notions of antiderivative and area under the curve commonly taught in mathematics courses.

\section{Summations of differential products}

The findings above show that student success in physics integration is at least partially tied to an understanding of the differential, which may lead to success in physics integration when viewed as an infinitesimal physical amount or interval, and differential products. But fundamentally, integration is an accumulation of such quantities, and this notion of "summation" is frequently referenced in the existing literature. Introductory physics students may not share this view, despite recent studies discussing its value to contexts of physics and engineering [11]. Building on earlier work by Meredith and Marrongelle [2], Doughty, McLoughlin, and van Kampen investigated the cues that coax students to attempt an integral solution in an intermediate electromagnetism course [3]. Even at the postintroductory level, they found that only 5\% of student participants demonstrated beliefs consistent with integration as a summation; their results suggested that, on the contrary, the predominant view of integration was merely an evaluation technique. Their results led to instructional changes to introduce and highlight integration as an infinite summation.

To emphasize the understanding of each of these two independent aspects of integral structure (the product and the sum), Jones developed the multiplicatively based summation (MBS) conception, defined as (i) the product of the integrand and the differential to create a new quantity, and (ii) the notion of adding up small (or infinitesimally small) amounts of the resultant product through small (or infinitesimally small) intervals of the domain to accumulate a total quantity [10]. After further study, Jones found that students were unlikely to use the MBS perspective to make sense of physical integrals, even in particularly beneficial scenarios, such as pressure integrated over an area to obtain a total force, $\int_{A} P d A$

\section{The process-object layered framework for understanding physics integrals}

Von Korff and Rebello developed a process-object layered framework for understanding integrals (specifically in the context of physics) reminiscent of that by Sealey [12]. It offers a variety of instructional paths for guiding student learning towards understanding physics integrals. Their "network" of layers differs from Sealey's by also offering strictly "microscopic" (infinitesimal) layers, such as differentials and differential products, which can be summed to produce "macroscopic" (finite) results. They assert that physics students can be taught that "an integral is "the sum of many infinitesimally small quantities,' without harm to their understanding".

According to Von Korff and Rebello's model, along the microscopic path to understanding integrals, the differential product lies between the differential and integral (e.g., $d t \rightarrow v d t \rightarrow \int v d t$ ). In our study, we hypothesize that this chain suggests that conceptual understanding of differential products "mediates" understanding of integrals. For a discussion of regression-based mediation; see Sec. II D. Although probably intuitive and credible to experts, the veracity of such a relationship has not been quantitatively established among a population of student participants, and its potential existence could pose implications for physics integration instruction. In the next section we describe how mediation may be formally defined and measured.

\section{B. Primary research objective}

If mediation exists along the microscopic path of the physics integration layered framework by Von Korff and Rebello, students who better understand differentials (the independent variable) would see gains in differential product understanding as a result (as a mediator); likewise, knowledge gains in differential products would be associated with improvement in understanding of physics integrals (the dependent variable).

Therefore, this paper will examine quantitative evidence assessing the design of the "microscopic path" of the process-object layered framework for understanding physics integrals by Von Korff and Rebello. Specifically, we ask, "does the evidence reveal a mediating relationship between differentials and integrals, where the acting mediator variable is the understanding of differential products?"

\section{METHODS}

\section{A. Study participants}

Data from this experiment were obtained from two different student participant populations at the Ohio 
State University, a large research university. The first group of students were enrolled in the second semester of the standard calculus-based, introductory physics sequence ("Physics 2"). Students in this group received course credit for their participation in our research or an alternative assignment if they declined consent for the study. Over $80 \%$ of students enrolled in the course participated in at least some physics education research investigations; $N_{2}=65$ of those participants were randomly selected for this particular study. Each participant completed paper tasks in a quiet testing room for no more than $55 \mathrm{~min}$. Data were obtained for a duration of two weeks with approximately one-third of the semester remaining.

The second group of students were enrolled in the first semester of the standard calculus-based, introductory physics sequence ("Physics 1"). Research data was gathered from students at the end of the semester via an online assignment for course credit, with full credit for participation. While all enrolled students were required to complete the assignment from which the data was taken, only students who consented to the use of their results for research purposes were included in this study's data. In total, $N_{1}=1102$ students comprise the Physics 1 group. More than $90 \%$ of enrolled students chose to participate in this study. Self-reported data regarding prior mathematics courses indicate that $88 \%$ of Physics 1 participants have already completed a standard Calculus 1 course in a previous semester or in high school. Note that Calculus 1 is a corequisite course for Physics 1 at our institution; however, our data suggest that most students already have prior experience with calculus before enrolling in Physics 1. Major discipline data are not available for Physics 1 participants in this particular study, but prior studies of the same population at our institution have consistently shown that more than $70 \%$ of Physics 1 students self-report as engineering (of various types) or physics majors. Note that demographics are similar for the previously mentioned Physics 2 group.

Because of integration with course curriculum and the strictly online nature of this assignment, many more participants were utilized in the Physics 1 group compared to the Physics 2 group, which instead required physical attendance to complete a research activity. No time limit was imposed for completion of the research task for this group. Because these data were obtained from both Physics 1 and Physics 2 populations during the same semester, there is no overlap in participation across groups.

\section{B. Study and assessment design}

To establish relationships between introductory physics students' understanding of differentials, differential products, and integrals, two assessment instruments were developed and administered to each population. The two instruments were very similar; only the physical contexts were adjusted to best suit the physics topics relevant for
TABLE I. Categories and physical contexts of assessment items for each population. Each quantity in the Physics 1 and 2 columns corresponds to two assessment items: a physical interpretation question and an explicit units question, both employing identical physical scenarios. For examples of individual items, see Appendixes A and B.

\begin{tabular}{lcc}
\hline \hline Physical & & \\
quantity & Physics 1 & Physics 2 \\
\hline Differentials & $d t, d x, d M$ & $d t, d x, d q$ \\
Differential & $v d t, a d t, F d x$, & $v d t, a d t, q E d x, \lambda d x$, \\
products & $P d A, A d B$ & $\rho \pi R^{2} d x, A d B$ \\
Integrals & $\int v d t, \int a d t, \int F d x$, & $\int v d t, \int a d t, \int q E d x$, \\
& $\int P d A, \int A d B$ & $\int \lambda d x, \int \rho \pi R^{2} d x, \int A d B$ \\
\hline \hline
\end{tabular}

each course. The instruments consisted of two parts, with a total of 26 multiple choice items for Physics 1 and 30 multiple choice items for Physics 2. The full assessments are presented in the Supplemental Material [13].

The first half of the task consisted of "physical interpretation" items presenting a given symbolic representation of a differential, differential product, or integral (e.g., $d t$ ) and asking the student to identify the correct physical description (e.g., "An extremely short duration of time"). Note that we used such conceptual and physical descriptions as "extremely short" rather than "infinitesimally small" because, in interviews, we found that the latter phrase is often chosen because students remember seeing it elsewhere, such as in math class, but are rarely able to explain why they chose it. These "interpretation" questions varied in physical context, with both concrete and generic (“ $\int A d B$ ") contexts. Examples of assessment interpretation items are shown in Appendix A.

More specifically, the first half of the instrument began with three items to assess the ability to interpret differentials in three physical contexts; this was followed by five (Physics 1) or six (Physics 2) questions relating to differential products in various contexts; finally, students were given five (Physics 1) or six (Physics 2) questions pertaining to integrals in various contexts. Note that for each differential product item (e.g., vdt), there was a corresponding integral item (e.g., $\int v d t$ ). The physical contexts and symbolic representations from each question are shown in Table I.

The second half of the instrument consisted of "units" items. These items were identical to the items in the first half, only the students were asked to identify the correct units for a given differential, differential product, or integral. Several examples are displayed in Appendix B. The first half of the instrument was collected before the second half was administered to prevent the students from cross referencing, and perhaps changing, the answers from the first half and the second half.

Together, with the interpretations scores for each set of quantities, students were given a single score for each of 
differentials, differential products, and integrals categories, equally weighted across all physical contexts and interpretation or units items. For example, a student who correctly answered all differentials units questions, but incorrectly answered all differentials interpretations questions, would receive a differentials score of $50 \%$. There were two reasons for choosing this scoring method. The first is theoretical and conceptual: each assessment dimension (interpretations and units) represents an aspect of a conceptual understanding of the quantity, namely, "what kind of quantity it is" and "what it physically means." Both represent operational means of assessment of conceptual understanding of the symbolic representations. The second reason is empirical, namely, for a given quantity the scores between the two dimensions were highly correlated, thus supporting the validity of the scoring method, as is shown in the next section.

\section{Assessment validity and reliability}

The assessment instruments were developed via our prior work including several semesters of iterations of design, implementation and item analysis, student interviews, and adjustment, addition or removal of items based on the results. Physical contexts and quantities were chosen based on calculus expressions commonly found in introductory calculus-based physics courses, and student interviews verified the relevance of the expressions. Important distractors were derived from free response questions pertaining to interpretations of differentials and differential products, such as the incorrect notion that differentials are "rates" or "derivatives" [14]. In addition, other incorrect answer choices were produced from questions relating to interpretations of integrals, such as the misconception that the differential at the end of an integral is merely a label that contributes neither meaning nor units to the integrand [15].

In both Physics 1 and Physics 2 studies, the assessment instruments, as a total score as well as each individual category score, were found to be statistically reliable. In Table II, we display the reliability measure for differentials

TABLE II. Assessment reliability statistics. Cronbach's $\alpha$ is shown for both the Physics 1 and Physics 2 populations for each set of assessment items. Below each Cronbach's $\alpha$, we show the number of items for each physical quantity from both assessments.

\begin{tabular}{lll}
\hline \hline Physical quantity & Physics 1 & Physics 2 \\
\hline Differentials & 0.843 & 0.807 \\
& Items: 6 & Items: 6 \\
Differential products & 0.863 & 0.877 \\
& Items: 10 & Items: 12 \\
Integrals & 0.790 & 0.826 \\
& Items: 10 & Items: 12 \\
All & 0.913 & 0.909 \\
& Items: 26 & Items: 30 \\
\hline \hline
\end{tabular}

questions, differential products questions, integral questions, and all questions (combined) for both populations of participants. Within each set of questions pertaining to a single physical quantity (e.g., differential products), we find good internal consistency. Furthermore, no single assessment item causes noticeably different or outlying results if removed.

\section{Regression-based statistical mediation}

According to Baron and Kenny [16], "a given variable may be said to function as a mediator to the extent that it accounts for the relation between the predictor and the criterion." In simple mediation, a third variable exists as an explanatory mechanism or process that underlies an observed relationship between an independent variable $X$ and a dependent variable $Y$ (see Fig. 1). By definition, a mediator variable $M$ exists causally between these two variables, such that changes in $X$ affect $M$, which then affect $Y$. The following set of linear regression models is used to assess whether mediation exists for these variables via ordinary least squares regression:

$$
\begin{gathered}
\hat{Y}=i_{Y_{1}}+c X, \\
\hat{M}=i_{M}+a X, \\
\hat{Y}=i_{Y_{2}}+b M+c^{\prime} X .
\end{gathered}
$$

The symbols $i_{Y_{1}}, i_{M}$, and $i_{Y_{2}}$ are constants (or "intercepts") and $a, b, c$, and $c^{\prime}$ are the regression coefficients to be determined by ordinary least squares regression. These equations are visually represented by the diagrams in Fig. 1.

Baron and Kenny argued for a set of criteria to establish the existence of a mediating relationship between these three variables, which is frequently referred to as the
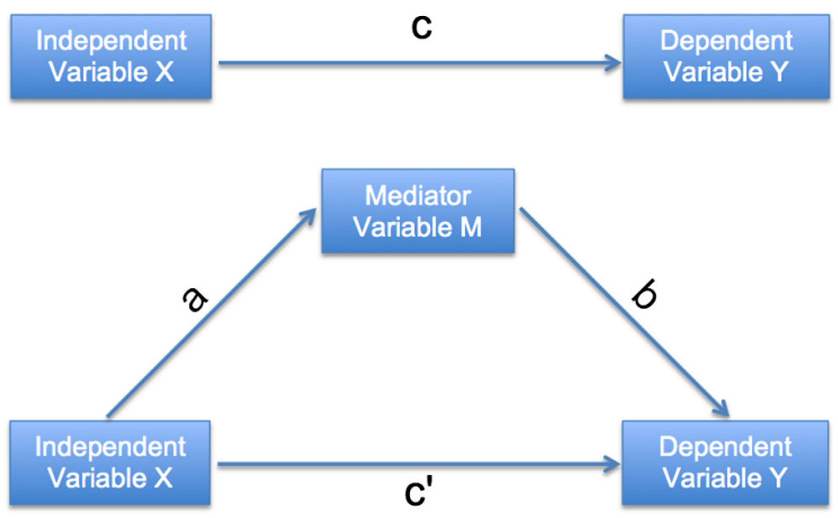

FIG. 1. Top: A visual representation of the total effect of a simple mediation model. Mathematically, this is shown in Eq. (1). Bottom: A visual representation of the indirect and direct effects of a simple mediation model. Mathematically, this is shown in Eqs. (2) and (3). 
"causal steps approach" [17]. We will describe this strategy to help intuitively explain mediation, but we will also discuss a more rigorous approach later in this section. In the causal steps approach, a significant "total effect" is obtained when a regression analysis returns a value for the coefficient $c$ in Eq. (1) that is statistically different from zero. This establishes an overall relationship between variables $X$ and $Y$. Next, a statistically significant value of $a$ in Eq. (2) suggests a relationship between the independent variable $X$ and the suspected mediator $M$. Finally, by Eq. (3), if the coefficient $b$ is significantly different from zero, while $c^{\prime}$ (known as the "direct effect") is closer to zero than $c$ (or statistically indistinguishable from zero), then mediation is said to exist via $M$ between $X$ and $Y$. The final step demonstrates the diminished direct relationship between $X$ and $Y$, while controlling for the effect of $M$.

This intuitive and straightforward recipe for determining the existence of mediation has been popular for several decades, but carries with it several drawbacks making it undesirable for use as an analysis tool in this paper. For an excellent argument, we recommend Hayes and Rockwood [17]. To summarize, philosophically, an empirical claim should be supported quantitatively by the effect most directly pertinent to that claim, particularly in as few inferential steps as necessary to minimize compounding uncertainty. In the case of mediation, establishing the existence of a nonzero "indirect effect" of $X$ on $Y$ through $M$ is sufficient; this is achieved by finding a statistically significant product of regression coefficients $a b$, which define the visual path from $X$ to $M$ to $Y$ as seen in Fig. 1 .

Furthermore, while the causal steps approach may still be informative in mediation analysis, it cannot convey an expression of confidence or uncertainty in the principal quantity of interest, the indirect effect $a b$. By contrast, the bootstrap resampling technique (or "bootstrapping") calculated by the PROCESS macro for the SPSS statistical software [18] can provide a confidence interval for the indirect effect [19]. Bootstrapping employs several thousand iterations of random sampling with replacement from an existing data sample to model inference about a population. Through successive resampling simulations, measures of accuracy can be obtained for sample estimates, including confidence intervals for the indirect effect $a b$ in mediation. Moreover, unlike other popular statistical methods, the reliability of bootstrapping is not contingent on normally distributed samples. This is a crucial benefit, as the sampling distribution of an indirect effect is not likely to be normal [20].

\section{RESULTS AND DISCUSSION}

\section{A. Descriptives statistics}

Descriptive statistics for each of the three scores (differentials, differential products, and integrals) for each population are displayed in Table III. Participants from the
TABLE III. Assessment descriptive statistics. Mean scores with 1 standard error are shown for both the Physics 1 and Physics 2 populations for each set of assessment items.

\begin{tabular}{lcc}
\hline \hline Physical quantity & $\begin{array}{c}\text { Physics 1 } \\
(N=1102)\end{array}$ & $\begin{array}{c}\text { Physics 2 } \\
(N=65)\end{array}$ \\
\hline Differentials & $47 \% \pm 1 \%$ & $73 \% \pm 4 \%$ \\
Differential products & $32 \% \pm 1 \%$ & $59 \% \pm 4 \%$ \\
Integrals & $51 \% \pm 1 \%$ & $71 \% \pm 3 \%$ \\
\hline \hline
\end{tabular}

Physics 2 population were seen to significantly outperform students from Physics 1 in all three sets of assessment items pertaining to each physical quantity: differentials, $F(11165)=31.4, p<0.001, d=0.81$; differential products, $F(11165)=50.5, p<0.001, d=0.89 ; \quad$ integrals, $F(11165)=32.5, p<0.001, d=0.79$. This difference could be explained by several factors, including population differences between Physics 1 and Physics 2 due to attrition from grades or major requirements, as well as mathematics experience and background. In any case, this result is presented for informative purposes only, and will not be a principal focus in the following sections.

A more interesting feature was observed upon analyzing Table III between rows. By inspection, it is clear that mean scores on the set of differential products questions were lower in both populations than differentials and integral scores, which were comparable within each course. In the absence of other competing factors, and with a significant indirect effect (that is large compared to the Direct Effect) from our hypothesized mediation model, one might reasonably predict that integral scores would be lower than (or at best equal to) differential products scores, which would be in turn lower than differential scores. This expected cascading effect of scores could be explained by the fact that many students may successfully demonstrate understanding of differentials, but only some subset of those students could successfully answer differential products questions, and even fewer could demonstrate success on all three topics. To the contrary, our results do not show this.

To verify this apparent contradiction of our expectation, a paired-samples $t$ test was performed using a withinstudent design on differential products scores and integral scores. From this, we observed that integral scores significantly exceeded differential products scores (in both populations of participants): Physics 1, $t(1101)=25.7$, $p<0.001, d=0.77$; Physics 2, $t(64)=3.2, p=0.002$, $d=0.40$.

This surprising result prompted us to consider instances in which students could correctly answer questions about an integral but not its corresponding differential product (within identical contexts) (e.g., correctly interpreting $\int_{t_{1}}^{t_{2}} v d t$ but not $v d t$, or correctly identifying the units of $\int_{x_{1}}^{x_{2}} F d x$ but not $F d x$, etc.). We found that this possible inconsistency with our proposed model occurred in $27 \%$ of 
responses from Physics 1 students, and $20 \%$ of responses from Physics 2 students. In other words, a fifth or more of student responses in both populations demonstrated conceptual understanding of an integral, but not its differential product. These results could be seen to run counter to our mediation model, at least for the case of zero or very small direct effects. In Sec. III C, we will present evidence supporting a possible explanation: for some contexts that are apparently common and familiar, students may be able to succeed on integral questions in spite of lower differential products performance.

\section{B. Mediation from differential products}

Student understanding of differential products (with variable name "DP") is hypothesized to act as a mediator between the understanding of differentials (" $D$ ") and integrals ("Int") in the context of introductory physics. To assess the existence of this mediation, the following linear regression models were selected, based on those shown in Eqs. (1)-(3):

$$
\begin{gathered}
\widehat{\mathrm{Int}}=i_{1}+c D \\
\widehat{\mathrm{DP}}=i_{2}+a D \\
\widehat{\mathrm{Int}}=i_{3}+b \mathrm{DP}+c^{\prime} D
\end{gathered}
$$

Using the PROCESS macro for the SPSS statistical software discussed in Sec. II D and $k=10000$ bias-corrected bootstrap samples, a statistically significant indirect effect was observed in both populations at the $\alpha=0.05$ level. For $N=1102$ participants in the Physics 1 group, we observed an indirect effect of $a b=(0.55) \times(0.53)=0.29,95 \% \mathrm{CI}$ [0.25, 0.33]. Similarly, for $N=65$ participants in the Physics 2 group, we observed an indirect effect of $a b=(0.60) \times(0.46)=0.27, \quad 95 \% \quad$ CI $\quad[0.14,0.48]$. Because of the bootstrap confidence intervals (neither of which include zero in their range), we are $95 \%$ confident that the indirect effect in both populations is greater than zero, and thus that mediation exists for this set of variables among both participant groups. The regression model coefficients for both populations are displayed in Table IV.

Among Physics 1 participants, due to large sample size, all regression coefficients were seen to be significant at the $\alpha=0.05$ level. From this, we identified a significant total effect $(c=0.38 \pm 0.02)$ and a significant direct effect $\left(c^{\prime}=0.09 \pm 0.02\right)$, although the latter is a considerably smaller effect than the indirect effect $(a b=0.29 \pm 0.02)$ mentioned above. Apparently, as suggested by these data, improvement in differentials understanding is not directly associated with large improvements in students' abilities to understand integrals, relatively speaking. Instead, there is strong evidence that increased performance on differentials
TABLE IV. Linear regression model coefficients. Means with 1 standard error are shown for both the Physics 1 and Physics 2 populations for each regression coefficient from Fig. 1.

\begin{tabular}{lcc}
\hline \hline Model coefficient & Physics 1 (Sig.) & Physics 2 (Sig.) \\
\hline$a$ & $0.55 \pm 0.02$ & $0.60 \pm 0.10$ \\
& $(p<0.001)$ & $(p<0.001)$ \\
$b$ & $0.53 \pm 0.03$ & $0.46 \pm 0.11$ \\
& $(p<0.001)$ & $(p<0.001)$ \\
$c$ & $0.38 \pm 0.02$ & $0.20 \pm 0.10$ \\
& $(p<0.001)$ & $(p=0.040)$ \\
$c^{\prime}$ & $0.09 \pm 0.02$ & $-0.07 \pm 0.11$ \\
& $(p<0.001)$ & $(p=0.521)$ \\
\hline \hline
\end{tabular}

is related to increased performance on differential products, which is thereby related to gains in integral score.

In Physics 2, the sample size was only 6\% of the former group. Consequently, we did not identify a significant direct effect $\left(c^{\prime}=-0.07 \pm 0.11\right)$; if one exists, there was insufficient statistical power to discover it. Therefore, we cannot conclude from this sample that improvement in differentials understanding has a significant direct relationship with integral understanding. However, a significant total effect $(c=0.20 \pm 0.10)$ was observed among Physics 2 participants. Again, it is the indirect effect $(a b=0.27 \pm 0.09)$ that dominates this model. The data suggest that differentials gains are associated with differential products gains; likewise, higher differential products scores are associated with higher integral scores.

In Fig. 2, we summarize our findings by displaying the visual representation of the model discussed above, including regression coefficients.

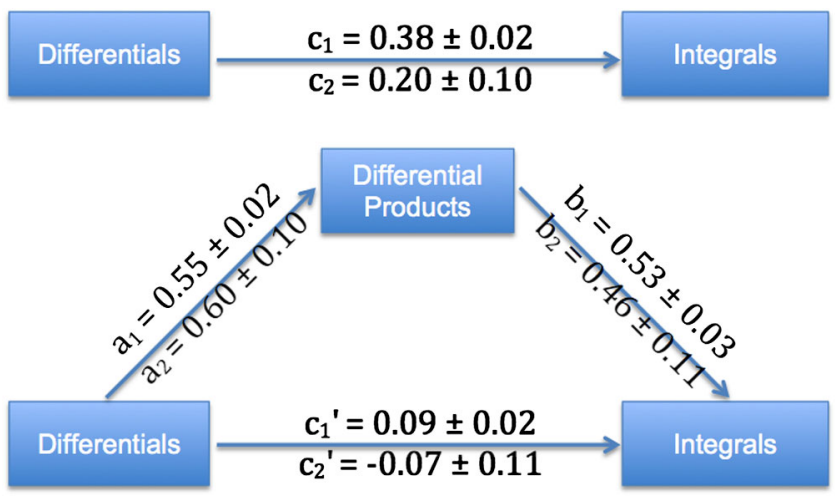

FIG. 2. Top: A visual representation of the total effect of our simple mediation model. The regression coefficient estimating the total effect is shown for both Physics 1 and Physics 2 populations as $c_{1}$ and $c_{2}$, respectively, with 1 standard error. Bottom: A visual representation of the direct and indirect effects of our simple mediation model. The regression coefficients are shown for both Physics 1 and Physics 2 populations with subscripts " 1 " and " 2 ," respectively, with 1 standard error. 


\section{Possible moderation from context familiarity}

During the analysis process, we observed that the indirect effects for several individual physical contexts appeared to differ (nominally, if not significantly) from each other depending on the scenario provided in the problem statement. The same linear regression models shown in Eqs. (4)-(6) can be used to compute the indirect effect for a single individual context featured on our assessment (see Table I), such as velocity vs time, in which the differential, differential product, and integral assessed were $d t, v d t$, and $\int v d t$, respectively. Among physical contexts with which students were likely very familiar (e.g., $\int v d t$ ), we generally observed lower indirect effects. Contrastingly, physical contexts that were presumed less familiar to students (e.g., $\int \rho \pi R^{2} d x$ ) tended to have slightly higher indirect effects. This information is summarized in Table V.

The notion that familiarity may play some role in how students arrive at their understanding of a physical integral is not unreasonable. After all, for a familiar context, students might access other resources to choose an answer instead of using the chain of reasoning from differentials to differential products to integrals studied here. For example, some students may simply directly recall from their current or past math and physics courses that the commonly used collection of symbols $\int v d t$ represents a displacement when written in that order, but they may not be able to correctly interpret the meaning of the differential product $v d t$ perhaps because it is less commonly encountered without the integral sign. This type of performance could weaken the indirect effect in contexts where familiarity is

TABLE V. Standardized indirect effects $a b$ estimated with 1 standard error for each model or context separately, where each model is of the form $d t \rightarrow v d t \rightarrow \int v d t$. Later, an independent or unrelated sample of early-semester Physics 2 students reported familiarity for each integral shown (" 1 " to "5," higher number indicates more familiar); familiarity is displayed in parentheses with 1 standard error.

\begin{tabular}{lcc}
\hline \hline Model or context & Physics 1 & Physics 2 \\
\hline$d t \rightarrow v d t \rightarrow \int v d t$ & $0.12 \pm 0.02$ & $0.12 \pm 0.09$ \\
& $(4.5 \pm 0.1)$ & $(4.5 \pm 0.1)$ \\
$d t \rightarrow a d t \rightarrow \int a d t$ & $0.13 \pm 0.02$ & $0.08 \pm 0.08$ \\
& $(4.4 \pm 0.1)$ & $(3.9 \pm 0.1)$ \\
$d x \rightarrow F d x \rightarrow \int F d x$ & $0.18 \pm 0.02$ & $0.24 \pm 0.10$ \\
& $(4.4 \pm 0.1)$ & $(3.3 \pm 0.1)$ \\
$d x \rightarrow \lambda d x \rightarrow \int \lambda d x$ & & $0.19 \pm 0.09$ \\
& & $(3.5 \pm 0.1)$ \\
$d x \rightarrow P d A \rightarrow \int P d A$ & $0.27 \pm 0.02$ & \\
& $(3.2 \pm 0.1)$ & \\
$d x \rightarrow \rho \pi R^{2} d x \rightarrow \int \rho \pi R^{2} d x$ & & $0.33 \pm 0.13$ \\
& & $(3.2 \pm 0.1)$ \\
\hline \hline
\end{tabular}

already sufficient for interpretation of the integrals, but not the differential products. On the other hand, some physical contexts and their respective integrals may be completely foreign to students, such as $\int \rho \pi R^{2} d x$. In this case, students would find more success comprehending the meaning of the differential product in order to make sense of the integral, which could strengthen the indirect effect for lessfamiliar questions.

In a later semester, we polled $N=106$ Physics 2 students in their first month of the course, that we might better understand prior experience and familiarity with the integrals assessed in our study from the perspective of introductory physics students. These students were not previously participants in our Physics 2 mediation study; however, their mean familiarity scores to the prompt, "How would you rank your familiarity with the following quantity? $(1=$ "Not at all familiar with this quantity" and $5=$ "Very familiar with this quantity")" were used to rank the student-perceived familiarity of the integrals used in the assessments. This between-students design is not suited to perform robust statistical tests on context as a moderating factor, but it can provide some qualitative insight into the role of familiarity as an influencing factor in our mediation model. We therefore display Fig. 3, which pairs mean selfreported familiarity of our assessment integrals with the indirect effects shown in Table $\mathrm{V}$, measured from the mediation study. Because of the large size of the error bars (each shown as one standard error), particularly among the Physics 2 mediation sample, broad claims cannot be made, but nominal indirect effect values generally align with our hypothesis that the indirect effect decreases with increasing familiarity.

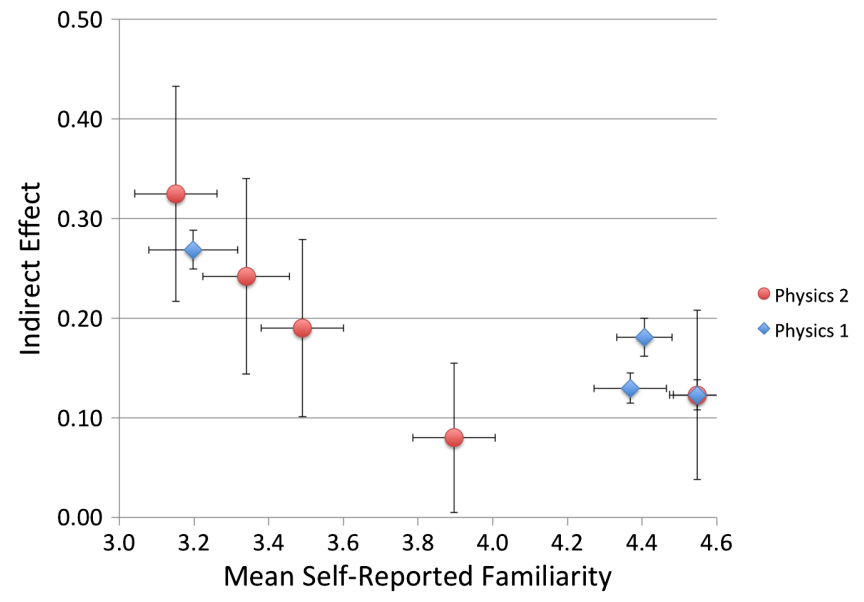

FIG. 3. Horizontal: Mean self-reported familiarity with each physical context from Table V. Vertical: Completely standardized indirect effect for each physical context from Table V. One standard error shown for both dimensions. Blue symbols represent contexts presented to Physics 1 students. Red symbols represent contexts presented to Physics 2 students. Note that familiarity scores were obtained from a separate sample of Physics 2 students in a later semester. 
TABLE VI. Percentage of students demonstrating conceptual understanding of most- and least-familiar differential products and integrals in both Physics 1 and Physics 2. In the most familiar context ( $\int v d t$ in both Physics 1 and Physics 2), students were more likely to make correct physical sense of the integral while failing to make sense of its differential product, when compared to the same task in the least familiar contexts ( $\int P d A$ in Physics 1 and $\int \rho \pi R^{2} d x$ in Physics 2). These data support the hypothesis that familiarity is being used to prop up correctness when possible, but when context is no longer accessible from memory or prior experience, students who are unable to demonstrate understanding of the differential product are less likely to demonstrate understanding of its corresponding integral.

\begin{tabular}{lcc}
\hline \hline Context & Physics 1 & Physics 2 \\
\hline$v d t$ & $25 \% \pm 1 \%$ & $60 \% \pm 4 \%$ \\
$\int v d t$ & $66 \% \pm 1 \%$ & $79 \% \pm 4 \%$ \\
$\begin{array}{l}\text { Correct } \int v d t \text { and } \\
\text { incorrect } v d t\end{array}$ & $44 \%$ of students & $25 \%$ of students \\
$P d A$ & & \\
$\int P d A$ & $25 \% \pm 1 \%$ & $\ldots$ \\
Correct $\int P d A$ and & $33 \% \pm 1 \%$ & $\ldots$ \\
$\quad$ incorrect $P d A$ & $14 \%$ of students & $\ldots$ \\
$\rho \pi R^{2} d x$ & & $52 \% \pm 5 \%$ \\
$\int \rho \pi R^{2} d x$ & $\ldots$ & $58 \% \pm 5 \%$ \\
Correct $\int \rho \pi R^{2} d x$ & $\ldots$ & $14 \%$ of students \\
and incorrect $\rho \pi R^{2} d x$ & & \\
\hline \hline
\end{tabular}

To further support the claim that high familiarity seems to reduce the role of differential products as a mediator in correctly answering the conceptual questions on integrals, we returned to the data described in Sec. III A from our mediation study to compare individual physical contexts in which students demonstrated conceptual understanding of a given integral, but not its differential product. These data are presented in Table VI.

As seen in Table VI, students were most capable of correctly answering integral questions about those contexts with which they were most familiar $\left(\int v d t\right.$ in both Physics 1 and Physics 2). But of particular importance, we note that they were most able to answer these integral questions even when unable to answer nearly identical different products questions, as long as the context was familiar. When the context was apparently most foreign ( $\int P d A$ in Physics 1 and $\int \rho \pi R^{2} d x$ in Physics 2), students were much less likely to make correct physical sense of the integral without the corresponding differential product.

In fact, we found that only $14 \%$ of Physics 2 responses were correct for $\int \rho \pi R^{2} d x$ items and simultaneously incorrect for $\rho \pi R^{2} d x$ items (rated the least familiar Physics 2 assessment context in our poll), whereas $25 \%$ of Physics 2 responses were correct for $\int v d t$ items and simultaneously incorrect for $v d t$ items (rated the most familiar Physics 2 assessment context in our poll). A chisquared test showed that this difference was significant at the $\alpha=0.05$ level for Physics 2 students: $\chi^{2}(4,65)=19.3$, $p=0.001$, with an effect size of (Cramér's) $V=0.39-\mathrm{a}$ large-sized effect.

Likewise, we also found that only $14 \%$ of Physics 1 responses were correct for $\int P d A$ items and simultaneously incorrect for $P d A$ items (rated the least familiar Physics 1 assessment context in our poll); but again, this was contrasted by $44 \%$ of Physics 1 responses that suggested understanding of $\int v d t$ without understanding $v d t$. Once more, a chi-squared test showed that this difference was significant at the $\alpha=0.05$ level for Physics 1 students: $\chi^{2}(4,1102)=37.8, p<0.001$, with an effect size of (Cramér's) $V=0.13$ - a small-to-medium-sized effect.

To summarize, our data illustrate that for the least familiar physical contexts, integral success is most paired with understanding of its corresponding differential product; the opposite (which is counter to our mediation model) appears to be true in the most familiar contexts.

In statistical terms, whereas mediation attempts to address how an independent variable $X$ affects a dependent variable $Y$, the hint of familiarity may be better understood as "moderation"-when and to what degree $X$ affects $Y$. A detailed discussion of moderation analysis is provided by Hayes and Rockwood [17]. The data presented in this paper do not permit a rigorous moderation analysis that might conclusively determine the effect of familiarity on our simple mediation model. Instead, a within-student design including familiarity ratings is needed. Nevertheless, our results do support the hypothesis that such moderation exists and warrants further study.

\section{CONCLUSIONS AND IMPLICATIONS FOR INSTRUCTION}

Von Korff and Rebello devised a process-object layered framework with a network of possible paths to understanding of physics integrals. Along the "microscopic path" (using differentials to build integrals; e.g., $d t \rightarrow v d t \rightarrow$ $\left.\int v d t\right)$, differential products are situated between differentials and integrals. We hypothesized that mediation via understanding of differential products may be present in the relationship between the assessment scores of differentials and integrals; this model is illustrated by Fig. 2. Among independent student populations (Physics 1 and Physics 2), our results are consistent with mediation via differential products understanding. Specifically, neither of the 95\% confidence intervals estimating the indirect effect $a b$ included zero; this is shown in Sec. III B.

If mediation exists along the microscopic path to understanding physics integrals in the manner suggested by our results, on average, students would find more success from instruction that follows the progression formulated by Von Korff and Rebello (i.e., $d t \rightarrow v d t \rightarrow \int v d t$ ). In particular, because of the very small (or possibly nonexistent) direct effect $\left(c^{\prime}\right)$ from Table IV, simply emphasizing the meaning 
and units of the differential may have no effect on students' ability to make sense of the integral. However, comprehension of physics differentials may prove beneficial in promoting understanding of the differential product, whose instruction may ultimately lead to better integral understanding.

We also find evidence that familiarity can play an important role in understanding integrals. Specifically, the results support the idea that for novel, unfamiliar integrals, instruction emphasizing the microscopic path mediated by understanding differential products may be an effective method. For more familiar integrals, it is clear that some students may have bypassed important understanding of the components of the integral while still having a general idea of the whole integral itself. This finding may also be useful for designing instruction, noting that the instructional method may benefit from adapting to the student familiarity with the integral of interest.

Finally, it is worth noting that ultimately, mediation models imply an assumed causal relationship. While our results are consistent with mediation via differential products understanding, we would also recommend a controlled experiment involving treatments in the form of instructional interventions to measure the indirect, direct, and total effects discussed in our simple mediation model. Such a study could probe the causal nature of the model, which was an assumption made in this study (in which all students were assessed on all topics without instructional intervention), as demonstrated by the direction of the arrows shown in Fig. 2.

\section{ACKNOWLEDGMENTS}

The authors would like to thank Alison Koenka and Andrew Hayes for their helpful comments on mediation models. Funding for this research was provided by the Center for Emergent Materials: an NSF MRSEC under Grant No. DMR-1420451.

\section{APPENDIX A: SAMPLE ASSESSMENT ITEMS: INTERPRETATION}

Examples of assessment interpretation questions are shown below. Each question presents participants with a physics differential, differential product, or integral to correctly interpret from several possible choices.

(1) A stopwatch is used to measure time $t$ (in seconds) as a car moves. Which of the following best describes $d t$ ?

(a) The time on the stopwatch at the moment the car starts moving.

(b) The rate (at a single moment) that the car's position changes as time passes.

(c) The rate (at a single moment) that time passes.

(d) An extremely small change in time.

(e) A change in time large enough to measure on the stopwatch.
(2) An electron in a magnetic field experiences an acceleration $a$ (in meters $/$ second $^{2}$ ) at time $t$ (in seconds). Which of the following best describes $a d t$ ?

(a) The acceleration of the electron at a single moment.

(b) The rate (at a single moment) the electron's acceleration is changing as time passes.

(c) The rate (at a single moment) the electron's velocity is changing as time passes.

(d) An extremely small change in the electron's velocity

(e) The velocity of the electron at a single moment.

(3) A car is traveling in a straight line. Between times $t_{1}$ and $t_{2}$ (in seconds), it has velocity $v$ (in meters/second). Which of the following best describes $\int_{t_{1}}^{t_{2}} v d t$ ?

(a) The total velocity of the car between $t_{1}$ and $t_{2}$.

(b) The total change in the car's position between $t_{1}$ and $t_{2}$.

(c) The average velocity of the car between $t_{1}$ and $t_{2}$.

(d) The total change in the velocity of the car between $t_{1}$ and $t_{2}$.

(e) The average position of the car between $t_{1}$ and $t_{2}$.

\section{APPENDIX B: SAMPLE ASSESSMENT ITEMS: UNITS}

Examples of assessment units questions are shown below. Each question presents participants with a physics differential, differential product, or integral whose units they must correctly identify from several possible choices.

(1) A stopwatch is used to measure time $t$ (in seconds) as a car moves. Which of the following shows possible units for $d t$ ?

(a) meters/second

(b) No units

(c) seconds/meter

(d) $1 /$ second

(e) seconds

(2) An electron in a magnetic field experiences an acceleration $a$ (in meters $/$ second $^{2}$ ) at time $t$ (in seconds). Which of the following shows possible units for $a d t$ ?
(a) meters/second ${ }^{2}$
(b) meters $/$ second $^{3}$
(c) meters/second
(d) meters
(e) No units

(3) A car is traveling in a straight line. Between times $t_{1}$ and $t_{2}$ (in seconds), it has velocity $v$ (in meters/ second). Which of the following shows possible units for $\int_{t_{1}}^{t_{2}} v d t$ ?
(a) No units
(b) meters/second
(c) meters
(d) meters $\cdot$ seconds
(e) meters $/$ second $^{2}$ 
[1] A. Orton, Students' understanding of integration, Educ. Studies Math. 14, 1 (1983).

[2] D. C. Meredith and K. A. Marrongelle, How students use mathematical resources in an electrostatics context, Am. J. Phys. 76, 570 (2008).

[3] L. Doughty, E. McLoughlin, and P. van Kampen, What integration cues, and what cues integration in intermediate electromagnetism, Am. J. Phys. 82, 1093 (2014).

[4] D.-H. Nguyen and N. S. Rebello, Students' difficulties with integration in electricity, Phys. Rev. ST Phys. Educ. Res. 7, 010113 (2011).

[5] D. Hammer, Student resources for learning introductory physics, Am. J. Phys. 68, S52 (2000).

[6] D. Hu and N. S. Rebello, Understanding student use of differentials in physics integration problems, Phys. Rev. ST Phys. Educ. Res. 9, 020108 (2013).

[7] A. Sfard, On the dual nature of mathematical conceptions: Reflections on processes and objects as different sides of the same coin, Educ. Studies Math. 22, 1 (1991).

[8] M. Zandieh, A theoretical framework for analyzing student understanding of the concept of derivative, Res. Collegiate Math. Educ. IV 8, 103 (2000).

[9] V. Sealey, A framework for characterizing student understanding of Riemann sums and definite integrals, J. Math. Behav. 33, 230 (2014).

[10] S. R. Jones, Areas, anti-derivatives, and adding up pieces: Definite integrals in pure mathematics and applied science contexts, J. Math. Behav. 38, 9 (2015).
[11] C. A. Manogue, K. Browne, T. Dray, and B. Edwards, Why is Ampère's law so hard? A look at middle-division physics, Am. J. Phys. 74, 344 (2006).

[12] J. Von Korff and N. S. Rebello, Teaching integration with layers and representations: A case study, Phys. Rev. ST Phys. Educ. Res. 8, 010125 (2012).

[13] See Supplemental Material at http://link.aps.org/ supplemental/10.1103/PhysRevPhysEducRes.14.010105 for a complete listing of all assessment items used in this study.

[14] N. Amos and A. F. Heckler, Proceedings of the Physics Education Research Conference 2015, College Park, MD (AIP, New York, 2015), pp. 35-38.

[15] R. M. Baron and D. A. Kenny, Proceedings of the Physics Education Research Conference 2014, Minneapolis, MN (AIP, New York, 2014), pp. 23-26.

[16] R. M. Baron and D. A. Kenny, The moderator-mediator variable distinction in social psychological research: Conceptual, strategic, and statistical considerations, J. Personality Social Psychol. 51, 1173 (1986).

[17] A. F. Hayes and N. J. Rockwood, Regression-based statistical mediation and moderation analysis in clinical research: Observations, recommendations, and implementation, Behav. Res. Therapy 98, 39 (2017).

[18] A. F. Hayes, Introduction to Mediation, Moderation, and Conditional Process Analysis: A Regression-Based Approach (The Guilford Press, New York, 2013).

[19] A. F. Hayes and M. Scharkow, Psychological Science 24, 1918 (2013).

[20] A. F. Hayes and M. Scharkow, The relative trustworthiness of inferential tests of the indirect effect in statistical mediation analysis, Psychol. Sci. 24, 1918 (2013). 